\title{
The representation of smooth functions in terms of the fundamental solution of a linear parabolic equation
}

\author{
by Neil A. Watson (Christchurch)
}

\begin{abstract}
Let $L$ be a second order, linear, parabolic partial differential operator, with bounded Hölder continuous coefficients, defined on the closure of the strip $X=$ $\left.\mathbb{R}^{n} \times\right] 0, a\left[\right.$. We prove a representation theorem for an arbitrary $C^{2,1}$ function, in terms of the fundamental solution of the equation $L u=0$. Such a theorem was proved in an earlier paper for a parabolic operator in divergence form with $C^{\infty}$ coefficients, but here much weaker conditions suffice. Some consequences of the representation theorem, for the solutions of $L u=0$, are also presented.
\end{abstract}

1. Introduction. Let $L$ be a second order, linear, parabolic partial differential operator, with bounded Hölder continuous coefficients (described more precisely below), defined on the closure of $\left.X=\mathbb{R}^{n} \times\right] 0, a[$. We prove a representation theorem for an arbitrary $C^{2,1}$ function, in terms of the fundamental solution of the equation $L u=0$. Such a formula in terms of the fundamental solution of Laplace's equation is classical [3, p. 11]. The result presented below was given, in the case where $L$ is the heat operator, by Smyrnélis [5] and, in a less natural form, by Doob [3, p. 271]. Subsequently, Watson [8] gave a proof for a parabolic operator in divergence form with $C^{\infty}$ coefficients. In the present note, the operator does not have to be in divergence form and the coefficients do not need to be so smooth. The techniques used here differ substantially from those in [8].

Some consequences of the representation theorem are also presented, and these again generalise the results in [8]. If $E$ is an open subset of $X, K$ is a compact subset of $E$, and $u$ is a solution of $L u=0$ on $E \backslash K$, we show that $u$ can be written uniquely in the form $u=v+w$, where $v$ is a solution of $L v=0$ on the whole of $E, w$ is a solution on $X \backslash K$, and $w$ vanishes both

2000 Mathematics Subject Classification: Primary 35K10.

Key words and phrases: representation theorem, parabolic equation, fundamental solution. 
at infinity and on $\left.\mathbb{R}^{n} \times\right] 0, k[$, where

$$
k=\inf \left\{t: K \cap\left(\mathbb{R}^{n} \times\{t\}\right) \neq \emptyset\right\} .
$$

This decomposition theorem permits an easy deduction of a general analogue of Bôcher's theorem from the particular case where $E$ is an infinite strip. Finally, in the case where $K$ is a subset of $\mathbb{R}^{n} \times\{b\}$, we give conditions on $u$ which ensure that $w$ is the integral of a signed measure against the fundamental solution of $L u=0$.

We consider the partial differential equation

$$
L u \equiv \sum_{i, j=1}^{n} a_{i j} D_{i} D_{j} u+\sum_{j=1}^{n} b_{j} D_{j} u+c u-D_{t} u=0
$$

where $D_{j} u=\partial u / \partial x_{j}$ for $j \in\{1, \ldots, n\}$ and $D_{t} u=\partial u / \partial t$. We assume that all the coefficients $a_{i j}, b_{j}, c$ are bounded continuous functions on $\bar{X}$, and that they satisfy a Hölder condition in $x$ uniformly with respect to $t$. We also assume that the coefficients $a_{i j}$ satisfy a Hölder condition in $t$ uniformly with respect to $x$, and that there is a positive constant $\lambda$ such that

$$
\sum_{i, j=1}^{n} a_{i j}(x, t) y_{i} y_{j} \geq \lambda\|y\|^{2}
$$

whenever $(x, t) \in \bar{X}$ and $y \in \mathbb{R}^{n}$. We assume, in addition, that the derivatives $D_{j} a_{i j}, D_{i} D_{j} a_{i j}, D_{j} b_{j}$ are bounded continuous functions on $X$ which satisfy a Hölder condition in $x$ uniformly with respect to $t$.

Under these conditions, the parametrix method can be used to construct a non-negative fundamental solution $\Gamma(x, t ; y, s)$ of $(1)$. Thus we obtain the function $\Gamma: X \times X \rightarrow \mathbb{R}$ which has the following properties:

(i) For each fixed point $(y, s) \in \bar{X}$, the function $\Gamma(\cdot, \cdot ; y, s)$ is a solution of (1) on $\left.\mathbb{R}^{n} \times\right] s, a\left[\right.$, and is zero on $\mathbb{R}^{n} \times[0, s]$.

(ii) For each continuous function $f$ on $\mathbb{R}^{n}$ with compact support, and each $s \in[0, a[$, we have

$$
\int_{\mathbb{R}^{n}} \Gamma(x, t ; y, s) f(y) d y \rightarrow f(\xi)
$$

as $(x, t) \rightarrow(\xi, s+)$ for any $\xi \in \mathbb{R}^{n}$.

(iii) There exist positive constants $C, c$ such that

$$
\Gamma(x, t ; y, s) \leq C(t-s)^{-n / 2} \exp \left(-\frac{c\|x-y\|^{2}}{t-s}\right)
$$

whenever $(x, t),(y, s) \in X$ and $s<t$. There is therefore a constant $K$ such that

$$
\int_{\mathbb{R}^{n}} \Gamma(x, t ; y, s) d y \leq K .
$$


(iv) As $t \rightarrow s+$,

$$
\int_{\mathbb{R}^{n}} \Gamma(x, t ; y, s) d y \rightarrow 1
$$

and, for any positive constant $\delta$,

$$
\int_{\|x-y\|>\delta} \Gamma(x, t ; y, s) d y \rightarrow 0 .
$$

(v) For each fixed point $(x, t) \in \bar{X}$, the function $\Gamma(x, t ; \cdot, \cdot)$ satisfies the equation adjoint to (1), namely

$$
L^{*} u \equiv \sum_{i, j=1}^{n} D_{i} D_{j}\left(a_{i j} u\right)-\sum_{j=1}^{n} D_{j}\left(b_{j} u\right)+c u+D_{t} u=0,
$$

on $\left.\mathbb{R}^{n} \times\right] 0, t[$.

Details can be found in [4].

The expressions $L u$ and $L^{*} v$ combine to give us Green's formula for $L$ :

$$
\begin{aligned}
& \int_{D}\left(v L u-u L^{*} v\right) d q \\
& \quad=\int_{\partial D}\left[\left(\sum_{j=1}^{n} a_{i j}\left(v D_{j} u-u D_{j} v\right)+\left(b_{i}-\sum_{j=1}^{n} D_{j} a_{i j}\right) v u\right) \nu_{i}-v u \nu_{t}\right] d \sigma,
\end{aligned}
$$

where $D$ is a bounded open subset of $X$ with a piecewise smooth boundary, $d q$ is the element of $(n+1)$-dimensional Lebesgue measure, $\left(\nu_{1}, \ldots, \nu_{n}, \nu_{t}\right)$ is the outward unit normal to $\partial D$, and $d \sigma$ is the element of surface area measure.

\section{The main result}

TheOREM 1. Let $E$ be a bounded open subset of $X$ with a piecewise smooth boundary, let $\left(x_{0}, t_{0}\right) \in E$, let $\Gamma_{0}=\Gamma\left(x_{0}, t_{0} ; \cdot, \cdot\right)$, and let $u \in C^{2,1}(\bar{E})$. Then

$$
\begin{aligned}
& u\left(x_{0}, t_{0}\right)=-\int_{E} \Gamma_{0} L u d q \\
& \quad+\int_{\partial E}\left[\left(\sum_{j=1}^{n} a_{i j}\left(\Gamma_{0} D_{j} u-u D_{j} \Gamma_{0}\right)+\left(b_{i}-\sum_{j=1}^{n} D_{j} a_{i j}\right) \Gamma_{0} u\right) \nu_{i}-\Gamma_{0} u \nu_{t}\right] d \sigma .
\end{aligned}
$$

Proof. For each $\beta>0$, we put $E_{\beta}=\left\{(x, t) \in E: t<t_{0}-\beta\right\}$. We apply Green's formula for $L$, with $D=E_{\beta}$ and $v=\Gamma_{0}$ (so that $L^{*} v=0$ on $E_{\beta}$, by (v)). Thus we obtain 


$$
\begin{aligned}
& \int_{E_{\beta}} \Gamma_{0} L u d q \\
& \quad=\int_{\partial E_{\beta}}\left[\left(\sum_{j=1}^{n} a_{i j}\left(\Gamma_{0} D_{j} u-u D_{j} \Gamma_{0}\right)+\left(b_{i}-\sum_{j=1}^{n} D_{j} a_{i j}\right) \Gamma_{0} u\right) \nu_{i}-\Gamma_{0} u \nu_{t}\right] d \sigma .
\end{aligned}
$$

We shall make $\beta \rightarrow 0$ to obtain the required result.

If $\chi_{\beta}$ denotes the characteristic function of $E_{\beta}$, then because $u \in C^{2,1}(\bar{E})$ and the coefficients of $L$ are bounded, we have $\left|\chi_{\beta} \Gamma_{0} L u\right| \leq \sup _{E}|L u| \Gamma_{0}$. Since $\Gamma_{0}$ is integrable (by (iii)), it follows that

$$
\lim _{\beta \rightarrow 0} \int_{E_{\beta}} \Gamma_{0} L u d q=\int_{E} \Gamma_{0} L u d q .
$$

Now consider the integral over $\partial E_{\beta}$. We put

$$
\partial E_{\beta}=(\partial E)_{\beta} \cup H_{\beta},
$$

where

$$
(\partial E)_{\beta}=\left\{(x, t) \in \partial E: t<t_{0}-\beta\right\}, \quad H_{\beta}=\left\{(x, t) \in \partial E_{\beta}: t=t_{0}-\beta\right\},
$$

and denote the integrand by $f$. Since $f$ is piecewise continuous on $\partial E$, and $f(x, t)=0$ whenever $t \geq t_{0}$, we have

$$
\lim _{\beta \rightarrow 0} \int_{(\partial E)_{\beta}} f d \sigma=\int_{\partial E} f d \sigma .
$$

There remains the integral over $H_{\beta}$, where $\nu_{i}=0$ for all $i \in\{1, \ldots, n\}$, $\nu_{t}=1$, and $d \sigma=d y$ is the element of $n$-dimensional Lebesgue measure. Therefore we have only to consider

$$
\int_{H_{\beta}} \Gamma_{0} u d y=\int_{H_{\beta}} \Gamma_{0}\left(u-u\left(x_{0}, t_{0}\right)\right) d y+u\left(x_{0}, t_{0}\right) \int_{H_{\beta}} \Gamma_{0} d y .
$$

Given $\varepsilon>0$, we choose a closed cylinder

such that

$$
S=\left\{(y, s):\left\|x_{0}-y\right\| \leq \delta, t_{0}-\delta \leq s \leq t_{0}\right\} \subseteq E
$$

$$
\left|u(y, s)-u\left(x_{0}, t_{0}\right)\right|<\varepsilon \quad \text { for all }(y, s) \in S .
$$

We put $B(\delta)=\left\{\left(y, t_{0}-\beta\right):\left\|x_{0}-y\right\| \leq \delta\right\}$. If $\beta<\delta$, then

$$
\begin{aligned}
\int_{H_{\beta}} \Gamma_{0} \mid u- & u\left(x_{0}, t_{0}\right) \mid d y \\
& =\left(\int_{B(\delta)}+\int_{H_{\beta} \backslash B(\delta)}\right) \Gamma_{0}\left|u-u\left(x_{0}, t_{0}\right)\right| d y \\
& \leq \varepsilon \int_{\mathbb{R}^{n}} \Gamma_{0}\left(y, t_{0}-\beta\right) d y+2 \sup _{E}|u| \int_{\left\|x_{0}-y\right\|>\delta} \Gamma_{0}\left(y, t_{0}-\beta\right) d y \\
& \leq K \varepsilon+o(1)
\end{aligned}
$$


as $\beta \rightarrow 0$, in view of (iii) and (iv). Hence

$$
\lim _{\beta \rightarrow 0} \int_{H_{\beta}} \Gamma_{0}\left(u-u\left(x_{0}, t_{0}\right)\right) d y=0 .
$$

Finally, it follows from (iv) that

$$
\begin{aligned}
u\left(x_{0}, t_{0}\right) \int_{H_{\beta}} \Gamma_{0} d y & =u\left(x_{0}, t_{0}\right)\left(\int_{B(\delta)}+\int_{H_{\beta} \backslash B(\delta)}\right) \Gamma_{0} d y \\
& =u\left(x_{0}, t_{0}\right)(1+o(1))
\end{aligned}
$$

as $\beta \rightarrow 0$. The result follows.

Corollary. If $u \in C^{2,1}(X)$ and has compact support in $X$, then

$$
u\left(x_{0}, t_{0}\right)=-\int_{X} \Gamma\left(x_{0}, t_{0} ; \cdot, \cdot\right) L u d q
$$

for each $\left(x_{0}, t_{0}\right) \in X$.

Proof. In Theorem 1 , we choose $E$ to contain both $\left(x_{0}, t_{0}\right)$ and the support of $u$.

Using this corollary, we can extend to the solutions of (1) the decomposition theorem proved in [8]. The proof is unchanged.

TheOREM 2. Let $E$ be an open subset of $X$, let $K$ be a compact subset of $E$, and let $u$ be a solution of (1) on $E \backslash K$. Then $u$ can be written uniquely in the form $u=v+w$, where $v$ is a solution of (1) on $E, w$ is a solution of (1) on $X \backslash K$, and $w$ is zero both at infinity and on $\left.\mathbb{R}^{n} \times\right] 0, k[$ for $k=$ $\inf \left\{t: K \cap\left(\mathbb{R}^{n} \times\{t\}\right) \neq \emptyset\right\}$.

Theorem 2 enables us to deduce the following analogue of Bôcher's theorem from the particular case of an infinite strip given in [2]. The proof follows that of Theorem 3 in [8], where references to related works can be found.

Theorem 3. Let $E$ be an open subset of $X$, let $\left(y_{0}, s_{0}\right) \in E$, and let $u$ be a solution of (1) on $E \backslash\left\{\left(y_{0}, s_{0}\right)\right\}$ that is bounded below on some cylinder $\left\{(y, s):\left\|y-y_{0}\right\|<r, s_{0}<s<t_{0}\right\}$. Then $u$ can be written uniquely in the form $u=v+\kappa \Gamma\left(\cdot, \cdot ; y_{0}, s_{0}\right)$, where $v$ is a solution of (1) on $E$, and $\kappa \in[0, \infty[$.

Again following the methods of [8], we can improve Theorem 3 in several directions. This necessitates the use of techniques from [7], and therefore the precise lower estimate of the fundamental solution established by Aronson in [1].

Following [7], we call a family $\mathcal{F}$ of closed balls in $\mathbb{R}^{n}$ an abundant Vitali covering of $\mathbb{R}^{n}$ if, for any $x \in \mathbb{R}^{n}$ and $\varepsilon>0, \mathcal{F}$ contains uncountably many balls centred at $x$ with radius less than $\varepsilon$. 
Given any open subset $D$ of $\mathbb{R}^{n+1}$ such that $D \cap\left(\mathbb{R}^{n} \times\{0\}\right) \neq \emptyset$, we put $D(0)=\left\{x \in \mathbb{R}^{n}:(x, 0) \in D\right\}$ and $D_{+}=D \cap X$.

TheOREM 4. Let $u$ be a solution of (1) such that

$$
u(x, t)=\int_{\mathbb{R}^{n}} \Gamma(x, t ; y, 0) d \mu(y)+v(x, t)
$$

for all $(x, t) \in D_{+}$, where $\mu$ is a signed measure concentrated on $D(0)$ and $v$ is a solution of $(1)$ on $D_{+}$with a continuous extension to 0 on $D(0) \times\{0\}$. Let $\mathcal{F}$ be an abundant Vitali covering of $\mathbb{R}^{n}$. If there is a signed measure $\nu$ concentrated on $D(0)$ such that

$$
\lim _{t \rightarrow 0+} \int_{A \cap V} u(x, t) d x=\nu(A \cap V)
$$

whenever $A, V \in \mathcal{F}, V \subseteq D(0)$, and $A \cap V \neq \emptyset$, then $\mu=\nu$.

Theorem 4, combined with results from [1] and [6], allows us to prove the following result.

TheOREM 5. Let $E$ be an open subset of $X$, and let $K$ be a nonempty compact subset of $E \cap\left(\mathbb{R}^{n} \times\{b\}\right)$. If $u$ is a solution of (1) on $E \backslash K$ such that

$$
\liminf _{t \rightarrow b+} \int_{U} u^{+}(x, t) d x<\infty
$$

for some relatively open subset $U$ of $\mathbb{R}^{n}$ such that $K \subseteq U \times\{b\}$, then there exist a unique solution $v$ of (1) on $E$, and a unique signed measure $\mu$ supported in $K(b)=\left\{x \in \mathbb{R}^{n}:(x, b) \in K\right\}$, such that

$$
u(x, t)=v(x, t)+\int_{K(b)} \Gamma(x, t ; y, b) d \mu(y)
$$

for all $(x, t) \in E \backslash K$.

\section{References}

[1] D. G. Aronson, Non-negative solutions of linear parabolic equations, Ann. Scuola Norm. Sup. Pisa Cl. Sci. 22 (1968), 607-694.

[2] J. Chabrowski and N. A. Watson, Properties of solutions of weakly coupled parabolic systems, J. London Math. Soc. 23 (1981), 475-495.

[3] J. L. Doob, Classical Potential Theory and its Probabilistic Counterpart, Grundlehren Math. Wiss. 262, Springer, 1984.

[4] A. M. Il'in, A. S. Kalashnikov and O. A. Oleŭnik, Linear equations of the second order of parabolic type, Uspekhi Mat. Nauk 17 (1962), no. 3, 3-146 (in Russian); English transl.: Russian Math. Surveys 17 (1962), no. 3, 1-143.

[5] E. P. Smyrnélis, Sur les moyennes des fonctions paraboliques, Bull. Sci. Math. 93 (1969), 163-173. 
[6] N. A. Watson, Uniqueness and representation theorems for parabolic equations, J. London Math. Soc. 8 (1974), 311-321.

[7] - Boundary measures of solutions of partial differential equations, Mathematika 29 (1982), 67-82.

[8] - , A decomposition theorem for solutions of parabolic equations, Ann. Acad. Sci. Fenn. Math. 25 (2000), 151-160.

Department of Mathematics and Statistics

University of Canterbury

Private Bag 4800

Christchurch, New Zealand

E-mail: N.Watson@math.canterbury.ac.nz

Reçu par la Rédaction le 28.6.2000 